\title{
Crystal Growth, Thermal, Mechanical and Optical Properties of a New Organic Nonlinear Optical Material: Ethyl P-Dimethylamino Benzoate (EDMAB)
}

\section{Natarajan ${ }^{\mathrm{a}}$, J. Kalyana Sundar ${ }^{\mathrm{b}}$, P. Selvarajan ${ }^{\mathrm{a}}$, M. Arivanandhan ${ }^{\mathrm{c}^{*} \text {, }}$ K. Sankaranarayanan', S. Natarajan', Y. Hayakawa ${ }^{\mathrm{c}}$}

\author{
${ }^{a}$ Department of Physics, Aditanar College of Arts and Science, Tiruchendur, India \\ ${ }^{\mathrm{b}}$ School of Physics, Madurai Kamaraj University, Madurai-625021, India. \\ ${ }^{\mathrm{c}}$ Research Institute of Electronics, Shizuoka University, Johoku 3-5-1, Hamamatsu, Japan. \\ ${ }^{\mathrm{d}}$ School of Physics, Alagappa University, Karaikudi-630003, India. \\ *Corresponding author: arivu_cz@yahoo.co.in
}

\begin{abstract}
An organic material, namely, ethyl p-dimethylamino benzoate was crystallized for the first time by solution growth technique using pure and mixed solvents. Growth kinetics and morphology changes with solvents were investigated based on solute-solvent interactions of pure and mixed solvents. An appropriate mixed solvent for high quality crystals with well-defined morphology is reported. The absence of solvent molecules and the presence of various functional groups of the grown sample were qualitatively confirmed by FTIR spectroscopic studies. Thermal properties of the grown sample were analyzed by TG and DTA analysis. Mechanical properties of the EDMAB crystal were investigated by micro hardness studies. Moreover, the grown crystal shows high transparency in the visible and near IR regions. The material shows relatively high SHG efficiency than that of KDP.
\end{abstract}

Keywords: Organic compounds; Crystal growth; - Optical properties

\section{INTRODUCTION}

Benzoic acid and its derivatives are more useful in bio-medical applications especially they are good inhibitors of influenza viruses [1]. Some of the benzoic acid derivatives, such as 4aminobenzoic acid, have been extensively reported in coordination chemistry, as bifunctional organic ligands, due to variety of their coordination modes [2]. Ethyl p-amino benzoate also known as benzocaine is one of the benzoic acid derivatives mainly used as a best local 
anaesthesia. Its low solubility renders it unsuitable for injections but its slow absorption from mucous-lined surfaces makes it safer than other local anaesthetics for ulcers and wounds $[3,4]$. Recently, it was identified as an organic nonlinear optical (NLO) material which shows high second harmonic generation than that of potassium dihydrogen phosphate (KDP), a well known inorganic NLO material [5]. In this series, Ethyl p-dimethylamino benzoate (EDMAB) is generally known as tertiary amines which are mainly used as a part of self curing two part system for dental/medical compositions comprising degradable copolymers which are suitable for use as root canal sealants, root canal filling materials, dental restorative materials, implant materials, bone cements and pulp capping materials [6]. Moreover, since the chemical structure (Fig.1) of the material has the $\pi$ - electron conjugated system attached with electron donor and acceptor groups at opposite end, it could be applicable for nonlinear optical applications [7].

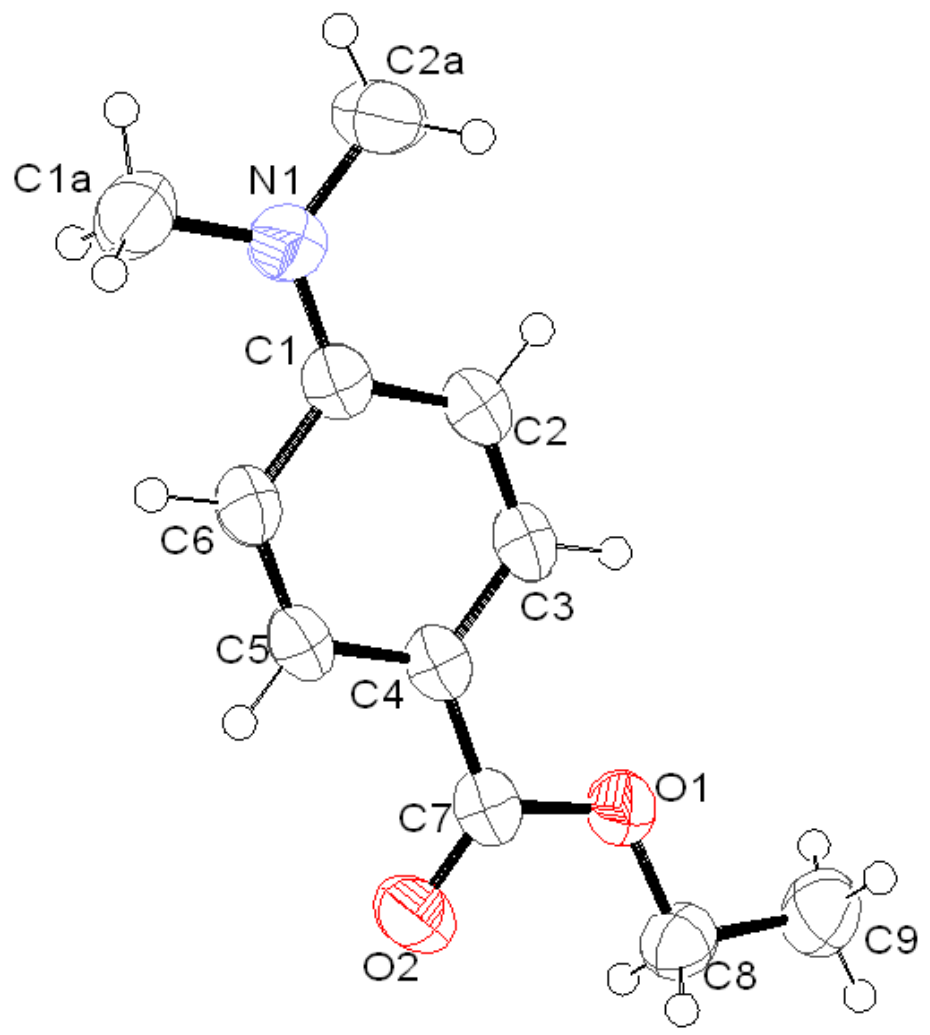

Figure 1: The molecular structure of the compound, showing the $30 \%$ probability displacement ellipsoids and the atom numbering scheme.

Recently, the crystal structure of EDMAB was solved by single crystal X-ray diffraction studies [8]. The EDMAB material crystallizes in the monoclinic system with the space group $\mathrm{P} 2{ }_{1} / \mathrm{a}$ and the lattice parameters are reported as $\mathrm{a}=12.695 \AA \hat{\mathrm{A}} \mathrm{b}=6.66 \AA$ and $\mathrm{c}=12.853 \AA$ and $\beta=98.672^{\circ}$ [8]. In the present work, large size EDMAB single crystals were grown by slow solvent evaporation method. The grown crystal was subjected to Fourier transform infrared studies to analyze the solvent incorporation during growth and ascertain various functional groups of the 
compound. Thermal properties of the material were investigated by means of thermo-gravimetric and differential thermal analysis.

\section{EXPERIMENTAL METHOD}

\subsection{Selection of the Solvent}

The EDMAB material was purchased from Aldrich and it was purified by recrystallization process using ethanol as a solvent. Organic crystal growth from solution mainly depends on the selection of a suitable solvent. Two major properties that would influence the crystal habit are assumed to be the difference in both the energy of crystallization and in the dipole moments between the crystallizing component and the solvent. If a solvent, which has similar dipole moment and the ideal solubility, is selected, large-size crystals can be expected to grow in the solution. On the other hand, a solvent has large differences in dipole moments from crystallizing substance and has poor solubility seems to be a poor solvent for the purpose of crystal growth with clear morphology. Most of the organic solvents have a dipole moment less than about 3 Debye. Unfortunately, organic dielectric materials are usually highly polar in nature so that the selection of solvent is restricted. The choice of solvent with a large dipole moment is likely to produce a crystal with a better habit growth.

Since EDMAB is insoluble in water, several organic solvents such as ethanol (1.69 Debye), methanol (1.70 Debye), toluene (0.38 Debye) and acetone (2.88 Debye) have been investigated to find the suitability for the single crystal growth of EDMAB. Among the above-mentioned solvents, it was observed that EDMAB has relatively low solubility in ethanol whereas it has relatively high solubility in acetone at $32^{\circ} \mathrm{C}$. In general, high solubility leads to uncontrolled spurious nucleation which hinders bulk single crystal growth. On the other hand, low solubility leads to less availability of growth units which restricts the size and growth rate of the crystal. Based on the preliminary observations made during the crystal growth experiments, conducted using all the above-mentioned solvents, ethanol and acetone were selected for further investigation towards attainment of large size and device quality crystals.

\subsection{Crystal Growth of EDMAB}

Solubility of EDMAB in mixed solvent of ethanol and acetone was measured at $32^{\circ} \mathrm{C}$ for different volume ratio of the solvents. Figure 2 shows the solubility of EDMAB in the mixed solvent as a function of acetone measuring in volume ratio. It can be seen from the figure that the solubility of the material increases evidently while increasing the volume ratio of acetone. According to the solubility data, saturated solution of EDMAB was prepared by dissolving the purified source material in the pure and mixed solvents of ethanol and acetone at $32^{\circ} \mathrm{C}$. The homogeneity of the solution was realized by continuous mild stirring of the solution using a 
magnetic stirrer without altering the solution temperature. Then, the near saturated solution was transferred to a crystallizer and covered by a perforated polyethylene sheet for controlled evaporation at room temperature. Transparent single crystals were harvested from the growth solution after achieved a reasonable size. Fig. 3a shows the photograph of the crystals grown from the mixed solvent of $25 \%$ acetone and $75 \%$ ethanol. The crystallization experiments conducted using the mixed solvents which contains $50 \%$ volume ratio and higher than that of acetone resulted uncontrolled large number of nucleation. Fig. $3 \mathrm{~b}$ shows one of the prepared specimen which was used for the following characterization studies.

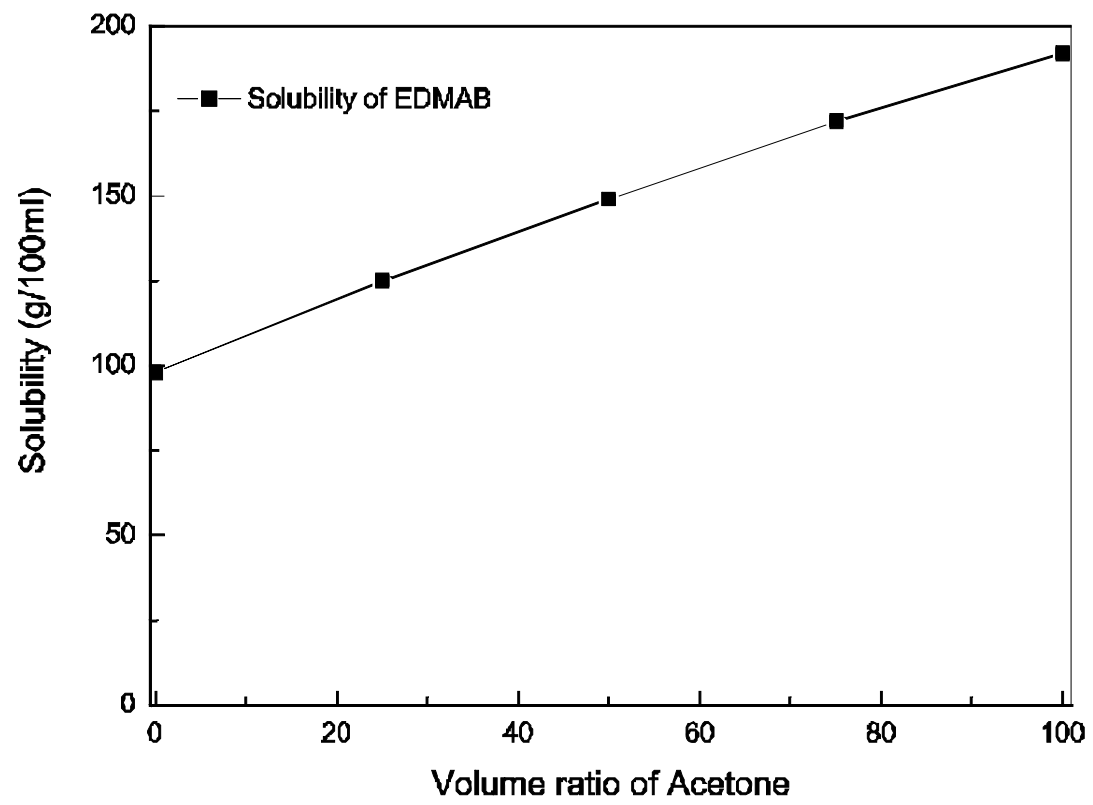

Figure 2: Solubility of EDMAB in mixed solvent of ethanol and acetone as a function of acetone volume ratio.

The physicochemical properties of the crystals grown from the mixed solvent ( $25 \%$ acetone and $75 \%$ ethanol) were studied by Fourier transform infrared spectroscopy (Perkin- Elmer), TG\&DTA analysis (STANTON REDCROFT), Vicker's microhardness tester and U-3000 double beam UV-VIS-NIR spectroscopy. As a preliminary study, the second harmonic generation of the grown crystal was confirmed be powder Kurtz method [9]. 
a

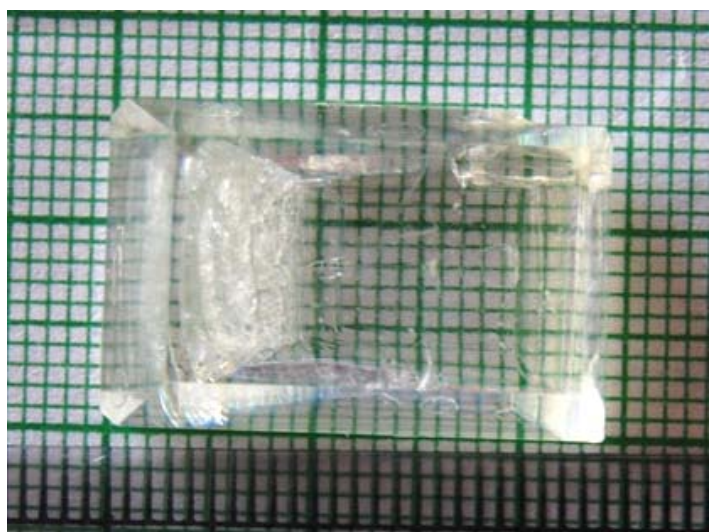

b

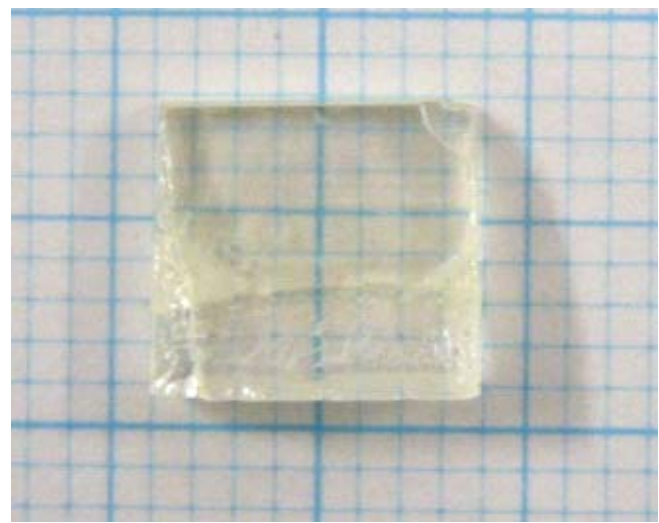

Figure 3: Photograph of the grown EDMAB crystal. (a). from the mixed solvent of ethanol and acetone of $75 \%$ and $25 \%$ volume ratio. (b). prepared specimen from the grown crystal

\section{RESULTS AND DISCUSSION}

In general, the growth rate of a growing crystal can largely be influenced by the size and rate of diffusion of molecules or growth units. In solution growth, the rate of diffusion of molecules or growth units are mainly depends on the solute-solvent interactions which can be effectively tuned by means of providing mixed solvents instead of single solvent. Due to the different vapour pressures of the constituent solvents as well as their interaction with solute molecules, the diffusion of growth units can very well be modified or controlled. Also, the vital growth parameters such as induction period and the nucleation rate can be varied by means of altering the chemical environment around the growing surface.

Based on these aspects, an attempt has been made to grow large size EDMAB crystals from mixed solvent containing ethanol and acetone, in which EDMAB has low solubility in ethanol and high solubility in acetone. Moreover, both the solvents are miscible and have different vapour pressures. This led us to investigate the controlling of supersaturation thereby nucleation of EDMAB, since changing the solubility as well as the interaction between the solute and the solvent has profound effect on these vital growth parameters. It was observed that the solubility of EDMAB was increased with the increasing of acetone volume ratio in the mixed solvents (Fig. 2). The results indicates that the effective interaction between the solute and solvent is higher when the dipole moment of the solvent increases. In the case of pure ethanol, due to the low dipole moment of the solvent, weak interaction with the solute was expected which has resulted low solubility. On the other hand, since the acetone has large dipole moment, strong interaction with solute molecules was expected which led to high solubility of the material. From the growth experiments, it was found that the mixed solvent contain $25 \%$ volume ratio of acetone yielded good quality large size $\left(25 \times 15 \times 8 \mathrm{~mm}^{3}\right)$ single crystals with short period (7days) of growth. The pure ethanol solvent system yielded thin platelet crystals with prolonged growth 
period and the mixed solvents with $\geq 50 \%$ volume ratio of acetone resulted spurious nucleation probably due to the relatively high vapour pressure of the solvent. The obtained large size EDMAB single crystal from the mixed solvent having $25 \%$ of acetone-volume ratio indicated an optimized growth condition. Moreover, the growth rate of EDMAB crystal along the a-axis seems to be much higher than that along b- and c-axes. Figure 4 shows the morphology of the grown crystal. Among the clearly observed facets, the crystal has two prominent flat facets such as $\{001\}$ and $\{00-1\}$ having large area compared to other $\{101\},\{010\}$ and $\{100\}$ faces.
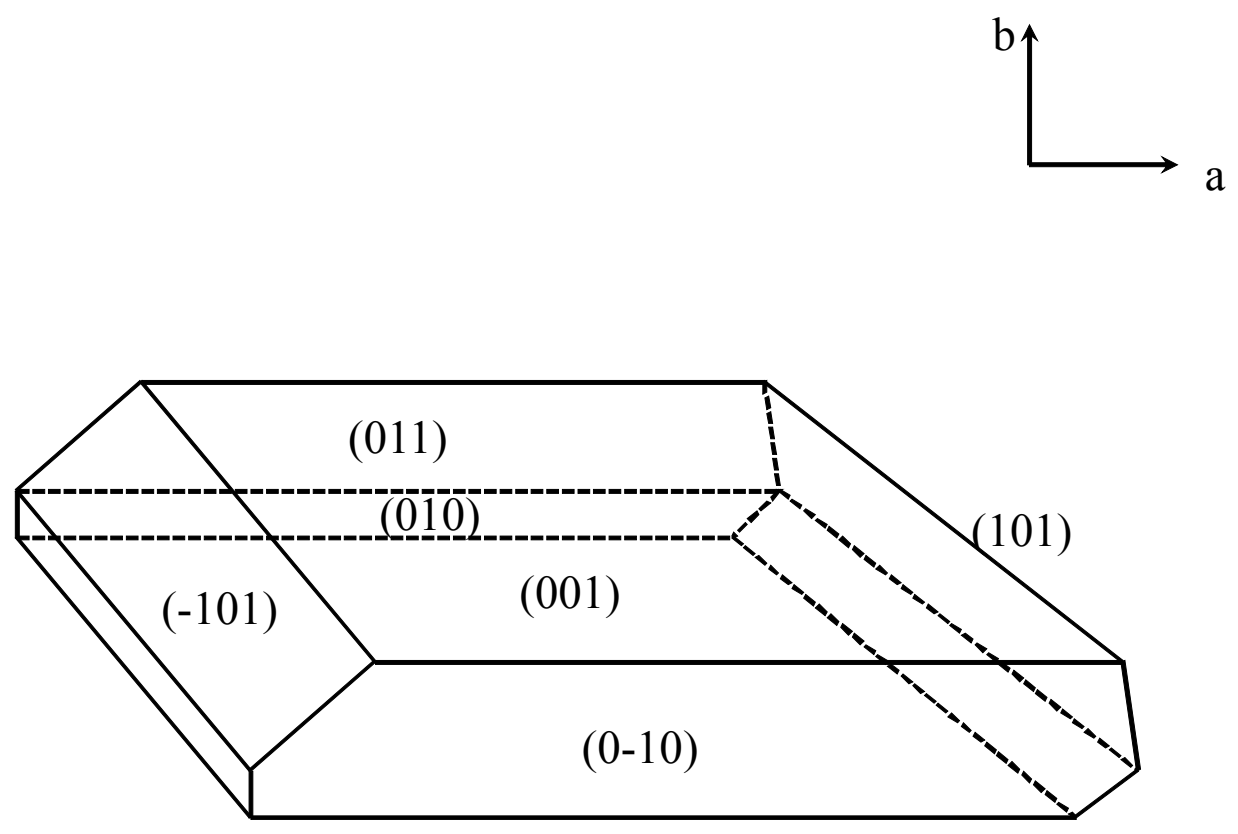

Figure 4: Morphology of the grown crystal.

\subsection{FTIR Studies}

The FTIR spectrum recorded in the range of $4000-400 \mathrm{~cm}^{-1}$ for EDMAB sample is shown in Figure 5. The various functional groups of the grown material were identified by FTIR spectroscopic analysis. $\mathrm{N}-\mathrm{H}$ is one of the prominent functional groups of the primary aromatic amines, and its asymmetric stretching vibration is observed at $3448 \mathrm{~cm}^{-1}$ and the symmetric stretching vibration is observed at $3392 \mathrm{~cm}^{-1}$. The aromatic $\mathrm{C}-\mathrm{H}$ stretching is observed at 3088 $\mathrm{cm}^{-1}$ and the aliphatic C-H stretching is observed at 2979, 2905 and $2812 \mathrm{~cm}^{-1}$. A strong peak

observed around $1697 \mathrm{~cm}^{-1}$ is due to the out of plane bending of $\mathrm{N}-\mathrm{H}$ vibration of the molecule. An absorption peak at $1605 \mathrm{~cm}^{-1}$ is due to carbonyl stretching $(\mathrm{C}=\mathrm{O})$ of the molecule. In plane 
bending of N-H stretching is observed at $1527 \mathrm{~cm}^{-1}$. The absorption peaks at 1482 and $1443 \mathrm{~cm}^{-1}$ are due to skeletal vibrations of aromatic ring. Out-of-plane bending vibration modes of aromatic $\mathrm{C}-\mathrm{H}$ bonds are observed at 1367, 1280, 1182, 1113 and $1012 \mathrm{~cm}^{-1}$. The absorption peaks observed below $1000 \mathrm{~cm}^{-1}$ illustrates in-plane-bending vibration modes of $\mathrm{C}-\mathrm{H}$ bonds. From this spectroscopic investigation, the presence of all the fundamental functional groups of the grown sample was confirmed qualitatively. Moreover, no absorption peaks related to the solvent molecules or any other impurities were observed which confirms the absence of solvent trapping in the crystal lattice and also the purity of the grown sample.

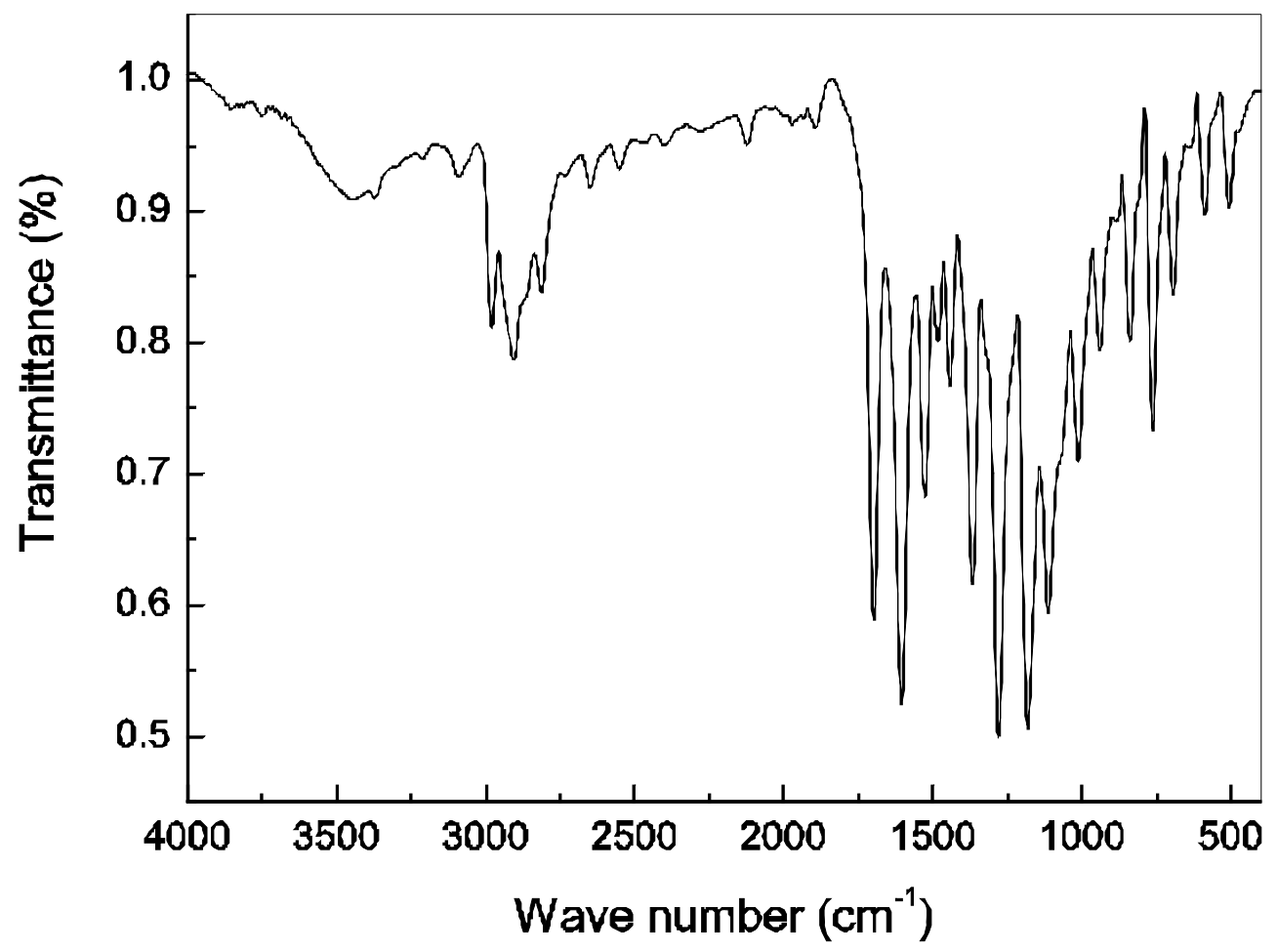

Figure 5: FTIR spectrum of the EDMAB crystal.

\subsection{TG \& DTA Studies}

Thermal characteristics of the material were investigated by TG \& DTA analysis. For the TG analysis, the known gram of material was heated from ambient temperature to $300^{\circ} \mathrm{C}$ at a heating rate of $20^{\circ} \mathrm{C} / \mathrm{min}$ in air atmosphere. Figure 6 illustrates the TG and DTA curves for the grown EDMAB sample. The TG curve shows very small $(2.5 \%)$ weight loss up to $127^{\circ} \mathrm{C}$. Hence the material is thermally stable up to $127^{\circ} \mathrm{C}$ and above this temperature the material looses its weight gradually. As can be seen from the DTA curve in the figure, the material undergoes an endothermic transition at $50.5^{\circ} \mathrm{C}$ where the melting begins. The endothermic peak at $64^{\circ} \mathrm{C}$ represents the temperature at which the melting terminates which corresponds to melting point of 
the material. The TG curve shows no weight loss during the first two endothermic peaks which confirms the phase transition of the material from solid to liquid (melting) and the weight of the material starts to losses after $128^{\circ} \mathrm{C}$ is not due to self-degradation of EDMAB but merely its evaporation after melting. The endothermic peak at $228^{\circ} \mathrm{C}$ indicates a phase change from liquid to vapor state as evidenced from the huge loss of weight in TG curve.

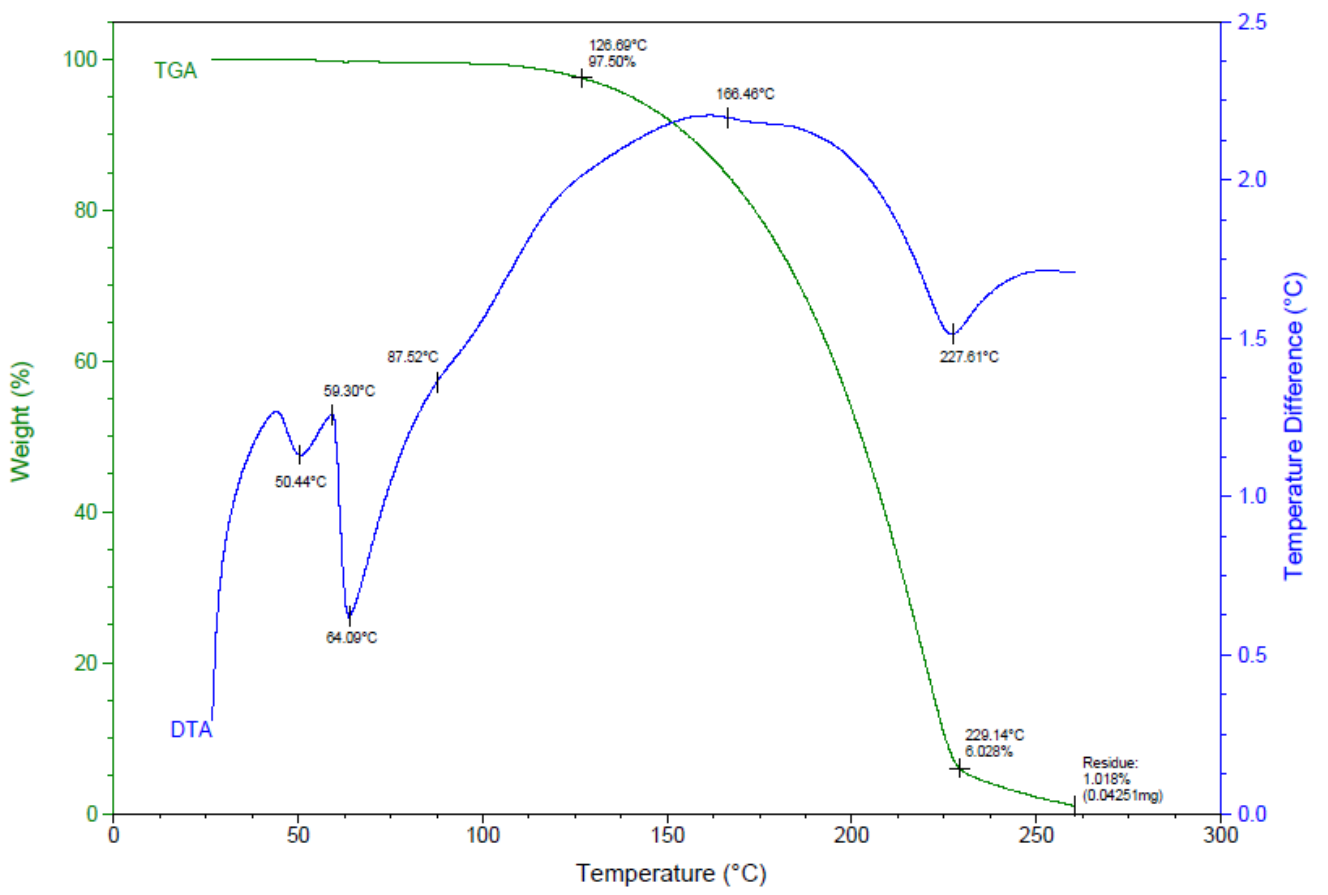

Figure 6: TG\&DTA curves of the grown EDMAB sample.

\subsection{Micro Hardness Studies}

Mechanical hardness of a material is also one of the decisive properties especially for post growth processes and device fabrications. In order to study the mechanical properties of the as grown EDMAB crystal, micro hardness was measured from 25 to 100 gram of load using HMV Microhardness tester. The indentation time was fixed as $10 \mathrm{~s}$ for all the samples. The diagonal lengths of the indented impression were measured for different loads varying from 25 to $100 \mathrm{~g}$. The successive indentations were made at different sites of the sample surface. The hardness values were calculated from the formula $\mathrm{Hv}=1.8544 \mathrm{P} / \mathrm{d}^{2} \mathrm{~kg} / \mathrm{mm}^{2}$, where $\mathrm{P}$ is the applied load and $\mathrm{d}$ is the mean diagonal length of the indentation. The variation of hardness as a function of applied load is shown in Figure 7 which reveals that the hardness increases with the increase of load. 


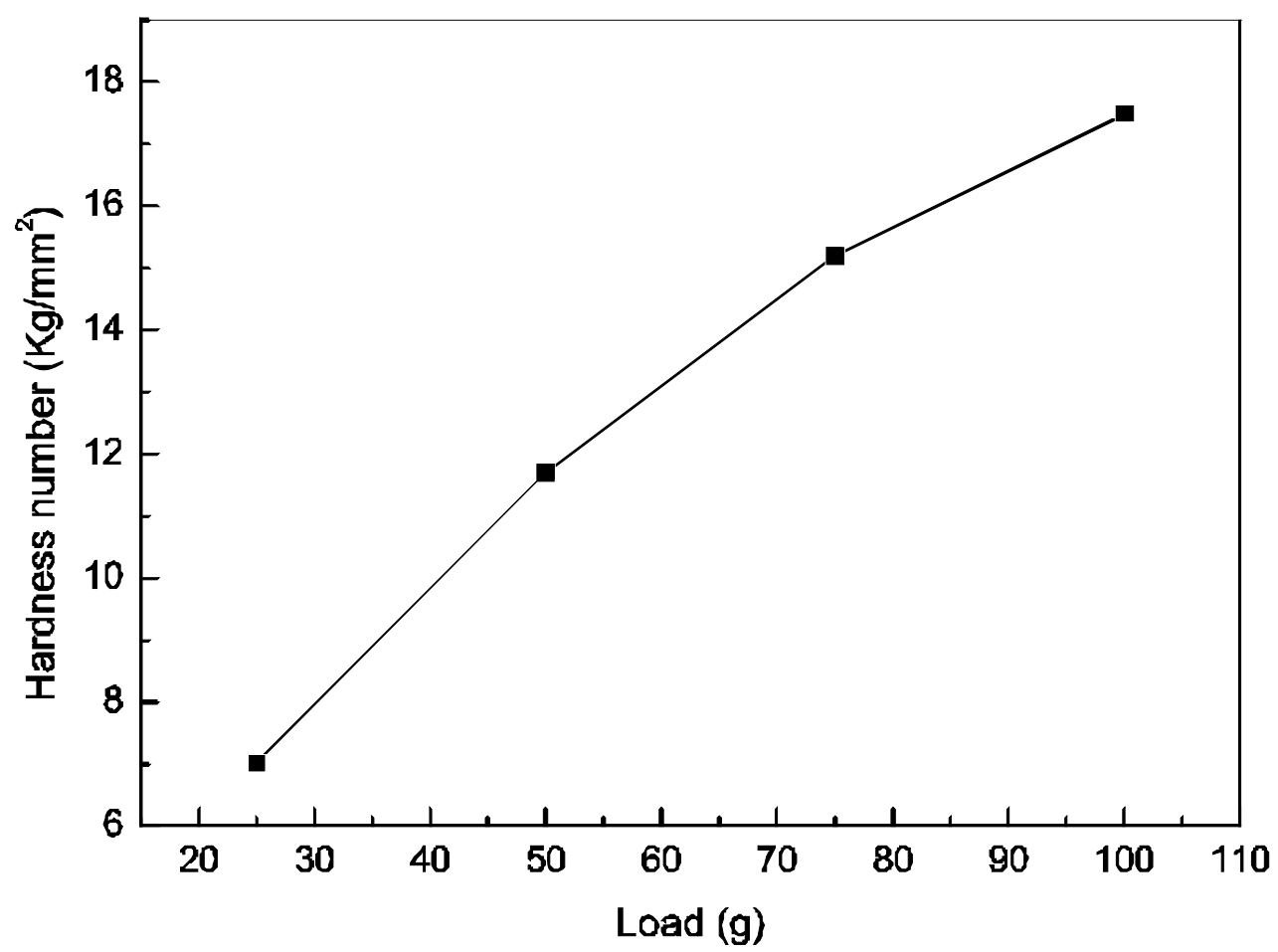

Figure 7: Load dependence of the hardness number of the EDMAB crystal

\subsection{Optical Studies}

The optical transmission spectrum was recorded in the wavelength range of $300-1100 \mathrm{~nm}$ at room temperature on the $\sim 3 \mathrm{~mm}$ thick sample prepared from the grown EDMAB crystal. The recorded transmittance spectrum is shown in Figure 8 . The material shows a wide transmission window in the wave length region of $400-1100 \mathrm{~nm}$. The sharp absorption onset at $340 \mathrm{~nm}$ and the high transmission values of the grown EDMAB single crystal at wavelength above $400 \mathrm{~nm}$ exhibit the low concentration of grown-in defects and high optical quality. 


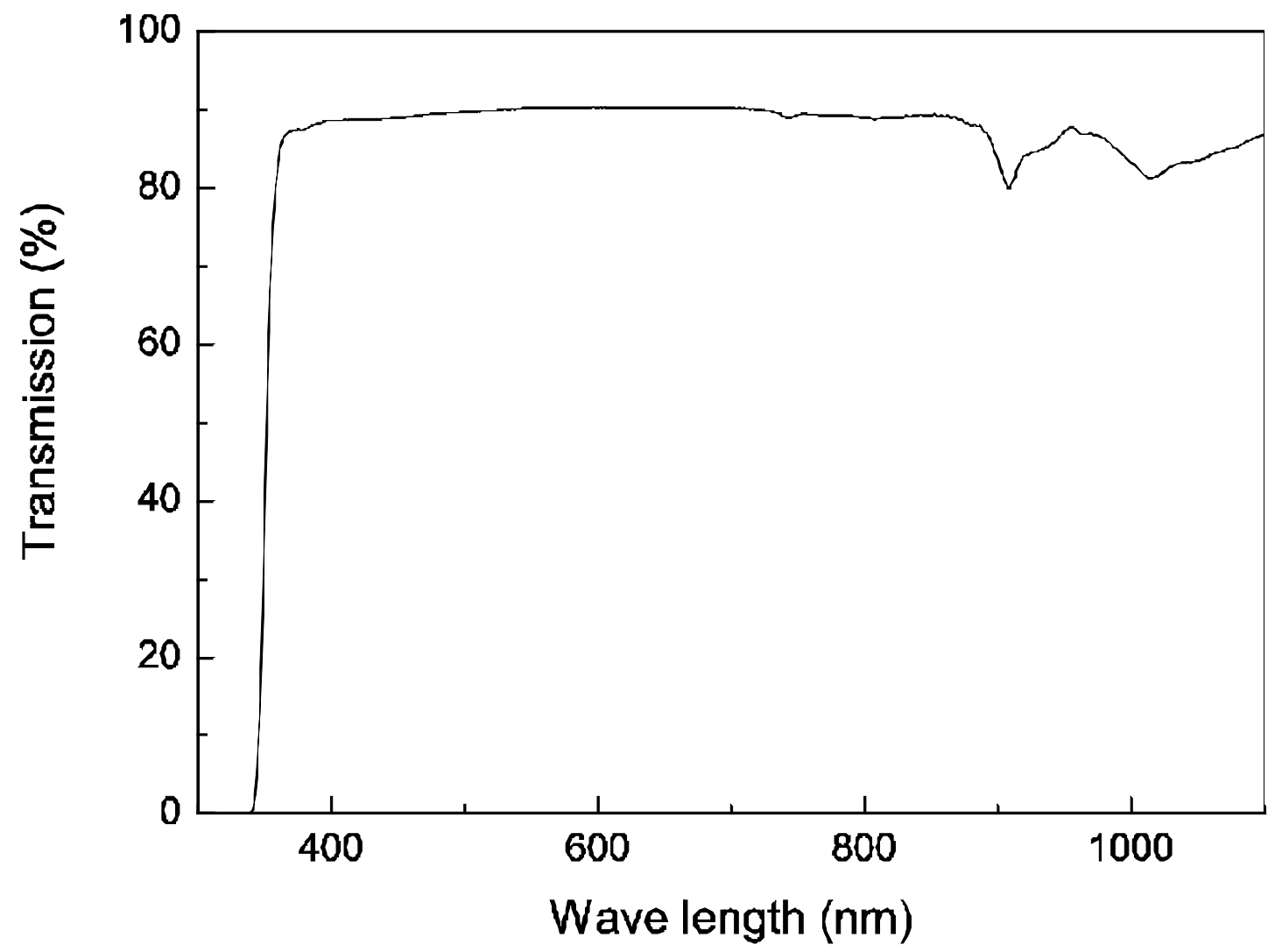

Figure 8: Optical transmission spectrum of the EDMAB sample.

\subsection{SHG Studies}

The SHG of the grown sample was confirmed by powder Kurtz method [9]. The powder sample prepared from the grown EDMAB crystal was packed in a triangular cell and kept in a cell holder. Nd:YAG laser of $1064 \mathrm{~nm}$ wavelength was used to irradiate the sample. Second harmonic signal was captured by the oscilloscope through the photomultiplier tube. Potassium dihydrogen phosphate (KDP) crystal was used as a reference material in the SHG measurement. The frequency conversion was confirmed by observing the green signal from the powdered EDMAB sample and the power of the SH signal is relatively higher (1.2 times) than that of pure KDP. Further investigations on phase matching studies of the grown crystal are under progress and the outcome will be published elsewhere.

\section{CONCLUSIONS}

EDMAB single crystals were grown by slow solvent evaporation technique using mixed solvent of ethanol and acetone. A mixed solvent having volume ratio of $75 \%$ ethanol and $25 \%$ acetone was found as a suitable solvent for bulk growth of EDMAB. Various functional groups present in the grown crystal were identified by FTIR spectroscopy. Thermal stability of the grown sample 
was studied by TG\&DTA analysis. TG curve of this sample indicates that the crystalline sample has a melting point $64^{\circ} \mathrm{C}$ and chemically stable up to $150^{\circ} \mathrm{C}$. Mechanical properties of the grown crystal were analyzed by micro hardness studies. The optical quality of the grown crystal was justified by optical transmission studies. The sharp absorption onset at $340 \mathrm{~nm}$ and the high transmission of the grown crystal at wavelength above $400 \mathrm{~nm}$ exhibit the optical quality, low concentration of grown-in defects and suitability for optical applications as well. The material shows high SHG efficiency than that of KDP and hence it could be applicable for nonlinear optical applications.

\section{REFERENCES}

1. M. Luo, M. J. Jedrzejas, S. Singh, C. L. White, W. J. Brouillette, G. M. Air, W. G. Laver, Acta Cryst. D51, (1995) 504-510.

2. R. Hauptmann, M. Kondo, S. Kitagawa, Z. Kristallogr. New Cryst. Struct. 215, (2000) 169172.

3. B. K. Sinha, V. Pattabhi, Proc. Indian Acad. Sci. Chem. Sci., 98, (1987) 229-234.

4. A. C. Schmidt, Pharm. Res. 22, (2005) 2121-2133.

5. M. Arivanandhan, K. Sankaranarayanan, P. Ramasamy, Materials Letters, 61, (2007) 4836.

6. Jia Weitao, Jin Shuhua United States Patent 6787584 (2004).

7. H. S. Nalwa, T. Watanabe, S. Miyata, in Nonlinear Optics of Organic Molecules and Polymers, Nalwa, H.S.; Miyata, S., Eds.; CRC press Inc.,: Florida, 1997; Chapter 4, pp 89350.

8. J. Kalyana Sundar, V. Natarajan, M. Arivanandhan, Y. Hayakawa, S. Natarajan, S, Acta Cryst. E, 66,(2010) 0355.

9. S. K. Kurtz, T.T. Perry, J. Appl. Phys. 39, (1968) 3798. 